PROCEEDINGS OF THE

AMERICAN MATHEMATICAL SOCIETY

Volume 128, Number 7, Pages 2121-2128

S 0002-9939(99)05459-3

Article electronically published on November 29, 1999

\title{
ISOMORPHISMS BETWEEN SUB-STRONGLY REDUCIBLE MAXIMAL TRIANGULAR ALGEBRAS
}

\author{
FANGYAN LU AND SHIJIE LU
}

(Communicated by David R. Larson)

\begin{abstract}
In this paper, we introduce the concept of sub-strongly maximal triangular algebras which form a large class of maximal triangular algebras, and prove that every algebraic isomorphism of sub-strongly maximal triangular algebras is spatially implemented, which generalizes the result by Ringrose in two respects.
\end{abstract}

\section{INTRODUCTION AND PRELIMINARIES}

Let $\mathcal{H}$ be a complex Hilbert space and $B(\mathcal{H})$ the set of all bounded operators on $\mathcal{H}$. Following [5], a subalgebra $\mathcal{S}$ of $B(\mathcal{H})$ is called a triangular algebra if $\mathcal{D}=\mathcal{S} \cap \mathcal{S}^{*}$ is a maximal abelian self-adjoint subalgebra (masa) of $B(\mathcal{H})$, where $\mathcal{S}^{*}=\left\{S^{*} \mid S \in \mathcal{S}\right\} . \mathcal{D}$ is then called the diagonal of $\mathcal{S}$. A projection on $\mathcal{H}$ is said to be a hull of $\mathcal{S}$ if it is invariant under $\mathcal{S}$. Clearly each hull lies in the diagonal. By Zorn's Lemma, it is easily seen that every triangular algebra $\mathcal{S}$ is contained in a maximal triangular algebra with the same diagonal as $\mathcal{S}$. The set of hulls of a maximal triangular algebra is a nest called the hull nest. A maximal triangular algebra is said to be strongly reducible if its hull nest is maximal.

This paper is devoted to the isomorphisms between maximal triangular algebras. In 1966, J. R. Ringrose [10] proved that if $\Phi: \mathcal{T} \rightarrow \mathcal{S}$ is an algebraic isomorphism between strongly reducible maximal triangular algebras, which maps the diagonal of $\mathcal{T}$ onto the diagonal of $\mathcal{S}$, then $\Phi$ is spatial, namely, there is an invertible operator $T$ such that $\Phi=\operatorname{Ad} T$. Naturally, one can propose the following questions:

1) Can one remove the condition of preserving diagonals?

2) Can one weaken the condition of strong reducibility?

For example, the spatiality of algebraic isomorphisms between nest algebras does not depend on the condition of preserving diagonals. As to question 2), by the definition of strong reducibility, a strongly reducible maximal triangular algebra must have a lot of hulls. But there do exist many triangular algebras which do not have many hulls. In fact, in this paper we introduce a new class of maximal triangular algebras, called sub-strongly reducible maximal triangular algebras, which properly contains the class of strongly reducible maximal triangular algebras, and proved that an algebraical isomorphism between sub-strongly reducible maximal

Received by the editors January 6, 1998 and, in revised form, September 4, 1998.

2000 Mathematics Subject Classification. Primary 47L75, 47L35.

Key words and phrases. Sub-strongly reducible maximal triangular algebras, hull nest, isomorphism. 
triangular algebras is spatial without the condition of preserving diagonals. Thus we generalized Ringrose's result in two respects.

This paper consists of three sections. In Section 2, we will introduce the concept of sub-strong reducibility, and prove that if $\Phi$ is an isomorphism from maximal triangular algebra $\mathcal{T}$ onto $\mathcal{S}$ and $\mathcal{S}$ is sub-strongly reducible, then so is $\mathcal{T}$. In Section 3 we will finally prove our main result.

In subsequent sections, isomorphisms always refer to algebraic isomorphisms.

\section{Sub-Strongly Reducible maximal triangular algebra}

Let $\mathcal{N}$ be a nest. For $N \in \mathcal{N}$, we define $N_{-}=\bigvee\left\{N^{\prime} \in \mathcal{N}: N^{\prime}<N\right\}$ and $N_{+}=\bigvee\left\{N^{\prime} \in \mathcal{N}: N^{\prime}>N\right\}$.

Definition. Let $\mathcal{N}$ be the hull nest of a maximal triangular algebra $\mathcal{S}$. $\mathcal{S}$ is a sub-strongly reducible maximal triangular algebra if $\operatorname{dim}\left((0)_{+} \ominus(0)\right) \leq 1$ and $\operatorname{dim}\left(\mathcal{H} \ominus \mathcal{H}_{-}\right) \leq 1$

Recall that a nest $\mathcal{N}$ is quasi-maximal if $N \ominus N_{-}$has dimension 0,1 or infinite for every $N \in \mathcal{N}$. Theorem 2 in [2] showed that given a quasi-maximal nest $\mathcal{N}$, one can construct a maximal triangular algebra with hull nest $\mathcal{N}$. Therefore sub-strongly reducible maximal triangular algebras form a large class of maximal triangular algebras.

Now we will give elementary properties of isomorphisms of the sub-strongly reducible maximal triangular algebras which are useful for our main result.

Let $x$ and $y$ be non-zero vectors in a Hilbert space $\mathcal{H}$. The rank one operator $x \otimes y$ is defined by $(x \otimes y) z=(z, y) x$ for each $z$ in $\mathcal{H}$.

Lemma 2.1. Let $E$ be a hull of a maximal triangular $\mathcal{T}$ and $x$ and $y$ two non-zero vectors.

(1) If $x \in E$ and $y \in E^{\perp}$, then $x \otimes y$ is in $\mathcal{T}$.

(2) If $x \in E$ and $y \in E_{-}^{\perp}$ and $\operatorname{dim}\left(E \ominus E_{-}\right) \leq 1$, then $x \otimes y$ is in $\mathcal{T}$.

Proof. (1) This is a consequence of Lemma 2.3.2 in [5].

(2) See the proof of Lemma 5.2 in [10.

Lemma 2.2. Let $\mathcal{T}$ be a sub-strongly reducible maximal triangular algebra and $T$ an element of $\mathcal{T}$. Then $T$ is a rank one operator if and only if the following condition is satisfied: if $A$ and $B$ are in $\mathcal{T}$ and $A T B=0$, then $A T=0$ or $T B=0$.

Proof. The necessity is obvious.

Sufficiency. The proof is a slight modification of the proof of Lemma 2.3 in [10]. We argue by contradiction. Let $\mathcal{N}$ be the hull nest of $\mathcal{T}$. Suppose that $T$ in $\mathcal{T}$ has rank at least two.

Case 1. $(0)_{+}=(0)$ and $\mathcal{H}_{-}=\mathcal{H}$. Then there are $N_{1}$ and $N_{2}$ in $\mathcal{N}$ with $(0)<N_{i}<\mathcal{H}(i=1,2)$ such that $\left.T\right|_{N_{1}}$ and $\left.S^{*}\right|_{N_{2}^{\perp}}$, where $S=T \mid N_{1}$, have rank at least two. Pick $x_{1}$ and $x_{2}$ in $N_{1}$ such that $N_{2}^{\perp} T x_{1}$ and $N_{2}^{\perp} T x_{2}$ are orthonormal. Take $0 \neq g \in N_{1}^{\perp}$ and $0 \neq h \in N_{2}$. Let $A=h \otimes N_{2}^{\perp} T x_{1}$ and $B=x_{2} \otimes g$. By Lemma 2.1. $A$ and $B$ belong to $\mathcal{T}$. But $A T B=0$ and $A T x_{1} \neq 0$ and $T B g \neq 0$.

Case 2. $(0)_{+} \neq(0)$ and $\mathcal{H}_{-}=\mathcal{H}$. Then there is $N$ in $\mathcal{N}$ with $(0)<N<\mathcal{H}$ such that $\operatorname{dim}[T N] \geq 2$. We choose vectors $x$ and $y$ in $N$ such that $T x$ and $T y$ are linearly independent. Let $f$ be a vector in $\mathcal{H}$ satisfying that $(T x, f) \neq 0$ and $(T y, f)=0$. Let $z$ be in $(0)_{+}$and $g$ in $N^{\perp}$. Define $A=z \otimes f$ and $B=y \otimes g$. 
By Lemma 2.1 and the sub-strongly reducibility of $\mathcal{T}, A$ and $B$ belong to $\mathcal{T}$. It is easily verified that $A T \neq 0 \neq T B$ and $A T B=0$.

Similarly, we can reach contradictions in the following two cases.

Case $3 .(0)_{+}=(0)$ and $\mathcal{H}_{-} \neq \mathcal{H}$.

Case $4 .(0)_{+} \neq(0)$ and $\mathcal{H}_{-} \neq \mathcal{H}$.

Thus we are done.

Theorem 2.3. Suppose that $\mathcal{T}$ and $\mathcal{S}$ are maximal triangular algebras and $\Phi$ is an isomorphism from $\mathcal{T}$ onto $\mathcal{S}$. If $\mathcal{S}$ is sub-strongly reducible, then $\Phi$ preserves rank one operators.

Proof. Let $x \otimes y \in \mathcal{T}$; then $\Phi(x \otimes y) \in \mathcal{S}$. Let $A, B \in \mathcal{S}$ and $A \Phi(x \otimes y) B=0$; then there exist $C$ and $D$ in $\mathcal{T}$ such that $A=\Phi(C), B=\Phi(D)$. Thus $\Phi(C x \otimes y D)=0$ and hence $C(x \otimes y) D=0$. Therefore $C(x \otimes y)=0$ or $(x \otimes y) D=0$. Equivalently $A \Phi(x \otimes y)=0$ or $\Phi(x \otimes y) B=0$. By the above lemma $\Phi(x \otimes y)$ is rank one.

Lemma 2.4. Let $\mathcal{T}, \mathcal{S}$ be maximal triangular algebras and $\Phi$ an isomorphism from $\mathcal{T}$ onto $\mathcal{S}$. For each $A$ in $\mathcal{T}$ or $\mathcal{S}$, let $P(A)$ be the orthogonal projection from $\mathcal{H}$ onto the closure of $A \mathcal{H}$. Then

(1) For each hull $E$ of $\mathcal{T}, P(\Phi(E))$ is a hull of $\mathcal{S}$ and $P\left(\Phi^{-1}(P(\Phi(E)))\right)=E$;

(2) $P(\Phi(\cdot))$ is an order preserving map from the hulls of $\mathcal{T}$ onto the hulls of $\mathcal{S}$;

(3) $P(\Phi(E))_{-}=P\left(\Phi\left(E_{-}\right)\right)$, for every hull $E$ in $\mathcal{T}$.

Proof. (1) This follows from part 2 of Lemma 2.9 in [10].

(2) Let $E<F$; then

$$
\Phi(E)=\Phi(F E)=\Phi(F) \Phi(E) .
$$

If $P(\Phi(E))>P(\Phi(F))$, then there is a vector $y$ such that $(1-\Phi(F)) \Phi(E) y \neq 0$. Then

$$
\Phi(E) y=(1-\Phi(F)) \Phi(E) y+\Phi(F) \Phi(E) y \neq \Phi(F) \Phi(E) y,
$$

contradicting (2.1). Thus we obtain $P(\Phi(E))<P(\Phi(F))$.

(3) We distinguish two cases.

Case 1. $E_{-}<E$. Then $P\left(\Phi\left(E_{-}\right)\right)<P(\Phi(E))$, hence $P(\Phi(E))_{-} \geq P\left(\Phi\left(E_{-}\right)\right)$. If $P(\Phi(E))_{-} \neq P\left(\Phi\left(E_{-}\right)\right)$, there is a hull $F$ of $\mathcal{S}$ such that

$$
P\left(\Phi\left(E_{-}\right)\right)<F<P(\Phi(E)) .
$$

Then by (1) we have

$$
E_{-}<P\left(\Phi^{-1}(F)\right)<E
$$

contradicting the definition of $E_{-}$.

Case 2. $E_{-}=E$. Then

$$
P(\Phi(E))_{-} \leq P\left(\Phi\left(E_{-}\right)\right)=P(\Phi(E))
$$

If $P(\Phi(E))_{-}<P\left(\Phi\left(E_{-}\right)\right)$, then by (1) there is a hull $E_{1}$ of $\mathcal{T}$ such that $E_{1}<E$ and $P\left(\Phi\left(E_{1}\right)\right)=P(\Phi(E))_{-}$. Since $E_{-}=E$, there is a hull $Q$ of $\mathcal{T}$ such that $E_{1}<Q<E$. Hence

$$
P(\Phi(E))_{-}=P\left(\Phi\left(E_{1}\right)\right)<P(\Phi(Q))<P(\Phi(E)),
$$

which contradicts the definition of $P(\Phi(E))_{-}$. Thus we are done. 
Lemma 2.5. Let $\mathcal{T}$ and $\mathcal{S}$ be maximal triangular algebras and $\Phi$ an isomorphism from $\mathcal{T}$ onto $\mathcal{S}$ which preserves rank one operators. If $E$ is a hull of $\mathcal{T}$ and $x \in E$, $y \in E_{-}^{\perp}$ with $x \otimes y \in \mathcal{T}$, then there are vectors $u$ and $v$ in $\mathcal{H}$ such that $u \in P(\Phi(E)$ ), $v \in P(\Phi(E))_{-}^{\perp}$ and $\Phi(x \otimes y)=u \otimes v$.

Proof. Since $\Phi$ preserves rank one operators, there is a rank one operator $u \otimes v \in \mathcal{S}$ such that

$$
\Phi(x \otimes y)=u \otimes v .
$$

Now we will show that $u$ and $v$ are desired.

By (2.2) we have that

$$
\Phi(x \otimes y)=\Phi\left((E x) \otimes\left(E_{-}^{\perp} y\right)\right)=\Phi(E) \Phi(x \otimes y) \Phi\left(E_{-}^{\perp}\right)=(\Phi(E) u) \otimes\left(\left(\Phi\left(E_{-}^{\perp}\right)\right)^{*} v\right) .
$$

Set $F=P(\Phi(E))$; then $F_{-}=P(\Phi(E))_{-}=P\left(\Phi\left(E_{-}\right)\right)$and hence $\Phi\left(E_{-}\right) F_{-}=F_{-}$. Thus for any $z \in F_{-}$, we have

$$
\begin{aligned}
\left(\Phi\left(E_{-}^{\perp}\right)^{*} v, z\right) & =\left(\left(1-\Phi\left(E_{-}\right)^{*}\right) v, F_{-} z\right) \\
& =\left(v,\left(F_{-}-\Phi\left(E_{-}\right) F_{-}\right) z\right)=\left(u,\left(F_{-}-F_{-}\right) z\right)=0 .
\end{aligned}
$$

Thus $\Phi\left(E_{-}^{\perp}\right)^{*} v \in F_{-}^{\perp}$. Since $v$ and $\Phi\left(E_{-}^{\perp}\right) v$ are linearly dependent, $v \in F_{-}^{\perp}$. Similarly $u \in F$.

By a similar argument we can obtain

Lemma 2.6. Let $\mathcal{T}$ and $\mathcal{S}$ and $\Phi$ be as in Lemma 2.5. IF $E$ is a hull of $\mathcal{T}$ and $x \in E, y \in E^{\perp}$, then there are vectors $u$ and $v$ in $\mathcal{H}$ such that $u \in P(\Phi(E))$, $v \in P(\Phi(E))^{\perp}$ and $\Phi(x \otimes y)=u \otimes v$.

Theorem 2.7. Let $\mathcal{T}, \mathcal{S}$ be maximal triangular algebras and $\Phi$ an isomorphism from $\mathcal{T}$ onto $\mathcal{S}$. If $\mathcal{S}$ is sub-strongly reducible, so is $\mathcal{T}$.

Proof. Suppose that $\mathcal{T}$ is not sub-strongly reducible. Then $\operatorname{dim}\left((0)_{+}^{\mathcal{T}} \ominus(0)^{\mathcal{T}}\right) \geq 2$ or $\operatorname{dim}\left(\mathcal{H}^{\mathcal{T}} \ominus \mathcal{H}^{\mathcal{T}}\right) \geq 2$, where $E_{+}^{\mathcal{T}}=\bigwedge\{F: F$ is a hull of $\mathcal{T}$ and $F>E\}$. Without loss of generality, assume that $\operatorname{dim}\left((0)_{+}^{\mathcal{T}} \ominus(0)^{\mathcal{T}}\right) \geq 2$. Take linearly independent vectors $x_{i}(i=1,2)$ in $(0)_{+}^{\mathcal{T}}$ and $y$ in $\mathcal{H}^{\mathcal{T}} \ominus(0)_{+}^{\mathcal{T}}$. Then by Theorem 2.3 and Lemma 2.6, there are vectors $u_{i}(i=1,2)$ in $(0)_{+}^{\mathcal{S}}$ and $v_{i}$ in $\mathcal{H}^{\mathcal{S}} \ominus(0)_{+}^{\mathcal{S}}$ such that

$$
\Phi\left(x_{i} \times y\right)=u_{i} \times v_{i}, \quad i=1,2 .
$$

Since $x_{1}$ and $x_{2}$ are linearly independent, $u_{1}$ and $u_{2}$ are also linearly independent which contradicts the sub-strongly reducibility of $\mathcal{S}$.

\section{ISOMORPHISMS OF SUB-STRONGLY REDUCIBLE MAXIMAL TRIANGULAR ALGEBRAS}

Lemma 3.1. Let $\mathcal{T}, \mathcal{S}$ be maximal triangular algebras and $\Phi$ an isomorphism from $\mathcal{T}$ onto $\mathcal{S}$ which preserves rank one operators. Let $E$ be a hull of $\mathcal{T}$ with $\operatorname{dim}(E \ominus$ $\left.E_{-}\right) \leq 1$. If $x_{i}(i=1,2)$ is a non-zero vector in $E$ and $y$ in $E_{-}^{\perp}$, then there are vectors $u_{i}$ and $v$ such that $\Phi\left(x_{i} \times y\right)=u_{i} \times v, i=1,2$.

Proof. By Lemma 2.1 $x_{i} \times y$ belongs to $\mathcal{T}$. Then there are vectors $u_{i}$ and $v_{i}$ such that

$$
\Phi\left(x_{i} \times y\right)=u_{i} \times v_{i}, \quad i=1,2 .
$$

Now it suffices to prove that $v_{1}$ and $v_{2}$ are linearly dependent. 
Let $\mathcal{D}$ be the diagonal of $\mathcal{T}$. By Lemma 17.4 of [1], there are $D_{i}$ in $\mathcal{D}$ and $x$ in $\mathcal{H}$ such that $D_{i} x=x_{i}$. We may assume $x \in E$ (if not, replace $x$ by $E x$ ). Let $\Phi(x \times y)=u_{3} \times v_{3}$. Thus, we have equations

$$
\Phi\left(x_{i} \times y\right)=\Phi\left(D_{i} x \otimes y\right)=\Phi\left(D_{i}\right) u_{3} \times v_{3}, \quad i=1,2 .
$$

Comparing (3.1) and (3.2), we are done.

By a similar argument as above, we can obtain

Lemma 3.2. Let $\mathcal{T}, \mathcal{S}$ be maximal triangular algebras and $\Phi$ an isomorphism from $\mathcal{T}$ onto $\mathcal{S}$ which preserves rank one operators. Let $E$ be a hull of $\mathcal{T}$. If $x_{i}(i=1,2)$ is a non-zero vector in $E$ and $y$ in $E^{\perp}$, then there are vectors $u_{i}$ and $v$ such that $\Phi\left(x_{i} \times y\right)=u_{i} \times v, i=1,2$.

Now our main result is in hand.

Theorem 3.3. Let $\mathcal{T}, \mathcal{S}$ be maximal triangular algebras and $\Phi$ an isomorphism from $\mathcal{T}$ onto $\mathcal{S}$. If $\mathcal{S}$ is sub-strongly reducible, then $\Phi$ is spatial.

Proof. By Theorem 2.7 $\mathcal{T}$ is also sub-strongly reducible. By Theorem 2.3 , $\Phi$ preserves rank one operators. We distinguish two cases.

Case 1. $\mathcal{H}^{\mathcal{T}}=\mathcal{H}_{-}^{\mathcal{T}}$. Then $\mathcal{H}^{\mathcal{S}}=\mathcal{S}_{-}^{\mathcal{S}}$.

Let $\mathcal{E}$ be the set of all hulls $E$ of $\mathcal{T}$ such that $E \neq(0)$ and $E<\mathcal{H}$, and let $\mathcal{F}$ be the set of those of $\mathcal{S}$. Assume given $E \in \mathcal{E}$ and a non-zero vector $y \in E^{\perp}$. Set $F=P(\Phi(E))$. By Lemmas 2.6] and 3.2, we obtain a bijective map $U_{E}$ from $E$ onto $F$ and vector $v$ in $F^{\perp}$ such that for any $x \in E$

$$
\Phi(x \otimes y)=U_{E} x \otimes v
$$

(see the proof of Theorem 3.1, [10] for details). Furthermore, we also have a bijective map $V_{E}$ from $E^{\perp}$ onto $F^{\perp}$ such that for any $x \in E$ and $y \in E^{\perp}$

$$
\Phi(x \otimes y)=U_{E} x \otimes V_{E} y .
$$

Now let $E$ be arbitrary. For each $E \in \mathcal{E}$, there are bijective maps $U_{E}$ from $E$ onto $F$ and $V_{E}$ from $E^{\perp}$ onto $F^{\perp}$ where $F=P(\Phi(E))$ such that

$$
\Phi(x \otimes y)=U_{E} x \otimes V_{E} y
$$

for all $x \in E$ and $y \in E^{\perp}$. Suppose $E_{1}$ and $E_{2}$ are in $\mathcal{E}$ with $E_{1}<E_{2}$. Then for each $x \in E_{1}$ and $y \in E_{2}^{\perp}$, we obtain

$$
U_{E_{1}} x \otimes V_{E_{1}} y=U_{E_{2}} x \otimes V_{E_{2}} y .
$$

Thus there are non-zero scalars $\eta$ and $\xi$ with $\eta \bar{\xi}=1$ such that

$$
U_{E_{2}}\left|E_{1}=\eta U_{E_{1}}, \quad V_{E_{1}}\right| E_{2}^{\perp}=\xi V_{E_{2}} .
$$

Pick up $E_{0}$ and replace $U_{E}$ and $V_{E}$ by scalar multiples so that they agree with $U_{E_{0}}$ and $V_{E_{0}}$ on their intersection. It follows that there is a map $U$ from $\bigcup\{E: E \in \mathcal{E}\}$ into $\bigcup\{F: F \in \mathcal{F}\}$ defined by $U \mid E=U_{E}$ for each $E$. Likewise, there is a map $V$ from $\bigcup\left\{E^{\perp}: E \in \mathcal{E}\right\}$ into $\bigcup\left\{F^{\perp}: F \in \mathcal{F}\right\}$ defined by $V \mid E^{\perp}=V_{E}$ for each $E$. Furthermore,

$$
\Phi(x \otimes y)=U x \otimes V y
$$

for every rank one operator in $\mathcal{T}$. 
Let $\mathcal{K}=\bigcup\{E: E \in \mathcal{E}\}$; then $\mathcal{K}$ is dense in $\mathcal{H}$. Furthermore, for any $x \in \mathcal{K}$ there is a non-zero vector $y$ such that $x \otimes y \in \mathcal{T}$. Thus for any $T \in \mathcal{T}$ we have

$$
\Phi(T) U x \otimes V y=\Phi(T x \otimes y)=U T x \otimes V y .
$$

Hence

$$
\Phi(T) U=U T
$$

holds on $\mathcal{K}$ for every $T \in \mathcal{T}$.

Now it suffices to prove that $U$ is bounded on $\mathcal{K}$. To do this, we need two claims.

Claim 1. $\sup \{\|U x\|: x \in E$ and $\|x\| \leq 1\}=M_{1}<\infty$.

It suffices to prove that $U$ is a closed operator with domain $E$.

Let $\left\{x_{n}\right\} \subset E$ and $x_{n} \rightarrow x$ and $U x_{n} \rightarrow y$. For every rank one operator $S \in \mathcal{S}$, $S U \in B(\mathcal{K}, \mathcal{H})$ since $U \Phi^{-1}(S) \in B(\mathcal{K}, \mathcal{H})$ and $S U=U \Phi^{-1}(S)$. Thus $S U x_{n} \rightarrow S y$ and $S U x_{n} \rightarrow S U x$. Hence we have that $S U x=S y$ for every rank one operator $S \in \mathcal{S}$.

(i) If $(0)_{+}^{\mathcal{S}}>(0)^{\mathcal{S}}$, we can choose a non-zero vector $u \in(0)_{+}$. Then for any $v$ in $\mathcal{H}$, by Lemma 2.1, $u \otimes v \in \mathcal{S}$. Therefore

$$
(u \otimes v) U x=(u \otimes v) y,
$$

which implies that $(U x, v)=(y, v)$ for all $v$ in $\mathcal{H}$. Thus $U x=y$. By the closed graph theorem, $U$ is bounded on $E$.

(ii) If $(0)_{+}^{\mathcal{S}}=(0)^{\mathcal{S}}$, then the span $\left\{Q^{\perp}: Q \in \mathcal{F}\right.$ and $\left.Q \neq(0)\right\}$ is dense in $\mathcal{H}$ in the norm-topology. For each $v \in \mathcal{H}$, there exists a sequence $\left\{v_{\beta} \in Q_{\beta}^{\perp}\right\}$ such that $v_{\beta} \rightarrow v$ in the norm-topology. Choose non-zero vectors $u_{\beta} \in Q_{\beta}$. Then since

$$
\left(u_{\beta} \otimes v_{\beta}\right) U x=\left(u_{\beta} \otimes v_{\beta}\right) y,
$$

we have $\left(U x, v_{\beta}\right)=\left(y, v_{\beta}\right)$ for each $\beta$, which implies that $(U x, v)=(y, v)$ for each $v$ in $\mathcal{H}$. Thus $U x=y$ and hence $U$ is bounded on $E$.

Claim 2. $\sup \left\{|(U x, v)|: x \in \mathcal{K},\|x\| \leq 1, v \in(P(\Phi(E)))^{\perp}\right.$ and $\left.\|v\| \leq 1\right\}=$ $M_{2}<\infty$.

Fix $u \in P(\Phi(E))$ and $v \in(P(\Phi(E)))^{\perp}$; then $u \otimes v \in \mathcal{S}$. Since $(u \otimes v) U=$ $U \Phi^{-1}(u \otimes v)$ and $U \Phi^{-1}(u \otimes v) \in B(\mathcal{K}, \mathcal{H})$, we have that $(u \otimes v) U \in B(\mathcal{K}, \mathcal{H})$. Therefore $\{(u \otimes v) U x: x \in \mathcal{K},\|x\| \leq 1\}$ is a bounded set; namely the set $\{(U x, v) u$ : $x \in \mathcal{K},\|x\| \leq 1\}$ is bounded for each $u \in P(\Phi(E))$ and $v \in(P(\Phi(E)))^{\perp}$. Hence by the uniform boundedness principle, Claim 2 is established.

Now by Claim 1, we have

$$
\|U P(E) x\| \leq M_{1}\|x\| \quad(x \in \mathcal{K}),
$$

and hence by (3.31)

$$
\|\Phi(E) U x\|=\|U P(E) x\| \leq M_{1}\|x\| \quad(x \in \mathcal{K}) .
$$

By Claim 2, since $\Phi(I-P(E))^{*} v \in(P(\Phi(P(E))))^{\perp}$ for each $v \in \mathcal{H}$, we have

$$
\begin{aligned}
\|\Phi(I-P(E)) U x\| & =\sup \{|(\Phi(I-P(E)) U x, v)|: v \in \mathcal{H} \text { and }|v| \leq 1\} \\
& =\sup \left\{\left|\left(U x,(\Phi(I-P(E)))^{*} v\right)\right|: v \in \mathcal{H} \text { and }|v| \leq 1\right\} \\
& \leq\|\Phi(I-P(E))\| M_{2}\|x\| \quad(x \in \mathcal{K}) .
\end{aligned}
$$

Thus for $x \in \mathcal{K}$ we have

$$
\|U x\| \leq\|\Phi(P(E)) U x\|+\|\Phi(I-P(E)) U x\| \leq\left(M_{1}+\|\Phi(I-P(E))\| M_{2}\right)\|x\|,
$$

that is, $U$ is bounded on $\mathcal{K}$. 
Case 2. $\operatorname{dim}\left(\mathcal{H}^{\mathcal{T}} \ominus \mathcal{H}_{-}^{\mathcal{T}}\right)=1$. Fix a non-zero vector $y$ in $\mathcal{H}^{\mathcal{T}} \ominus \mathcal{H}_{-}^{\mathcal{T}}$. Then by Lemmas 2.5 and 3.1 there are a bijective map on $\mathcal{H}$ and a non-zero vector $v$ in $\mathcal{H}^{\mathcal{S}} \ominus \mathcal{H}_{-}^{\mathcal{S}}$ such that

$$
\Phi(x \otimes y)=U x \otimes v
$$

for all $x$ in $\mathcal{H}$. Hence for all $T \in \mathcal{T}$ we have that

$$
\Phi(T) U=U T \text {. }
$$

Note that Claim 1 above holds for $E=\mathcal{H}$ in this case, therefore $U$ is bounded.

Thus the proof is completed.

The theorem above is the result about a certain type of triangular algebras but the triangularity of the algebra is not really used. The same proof can establish the following theorem.

Theorem 3.4. Let $\mathcal{N}_{i}(i=1,2)$ be a nest of a Hilbert space $\mathcal{H}_{i}$ and $\mathcal{T}_{i}$ a subalgebra of a nest algebra associated to $\mathcal{N}_{i}$ satisfying that

(1) $\mathcal{H}_{i} \ominus\left(\mathcal{H}_{i}\right)_{-}$and $\left(0_{i}\right)_{+} \ominus\left(0_{i}\right)$ both have dimension $\leq 1$;

(2) $\mathcal{T}_{i}$ contains a masa containing $\mathcal{N}_{i}$;

(3) The invariant subspace lattice of $\mathcal{T}_{i}$ is $\mathcal{N}_{i}$;

(4) $\mathcal{T}_{i}$ contains every rank one operator $x \otimes y$ such that $x \in E$ and $y \in E^{\perp}$ for some element $E$ of $\mathcal{N}_{i}$;

If $\Phi$ is an isomorphism from $\mathcal{T}_{i}$ onto $\mathcal{T}_{2}$, then $\Phi$ is spatially implemented.

Sketch of the proof. (i) Operators as in (2) of Lemma 2.1 are in $\mathcal{T}_{i}$ since $E-E_{-}$is in the masa and subtracting a scalar times $E-E_{-}$yields a rank one operator as in (4) above.

(ii) Let $T$ be in $\mathcal{T}_{i}$. The condition (1) asserts that the sufficient and necessary condition in Lemma 2.2 for $T$ to be rank one holds.

(iii) $\Phi$ preserves rank one operators by (ii).

(iv) $P(\Phi(\cdot))$ is an order isomorphism from $\mathcal{N}_{1}$ onto $\mathcal{N}_{2}$ since $P(\Phi(E))$ is in $N_{2}$ by $(3)$.

(v) Let $E$ (with $\operatorname{dim}\left(E \ominus E_{-}\right) \leq 1$ ) be in $\mathcal{N}_{1}$. If $x_{i}$ is a non-zero vector in $E$ and $y$ in $E^{\perp}\left(E_{-}^{\perp}\right)$, then there are vectors $u_{i}$ and $v$ such that $u_{i} \in P(\Phi(E))$, $v \in P(\Phi(E))^{\perp}\left(P(\Phi(E))_{-}^{\perp}\right)$ and $\Phi\left(x_{i} \otimes y\right)=u_{i} \otimes v$.

(vi) The rest is the same as that of Theorem 3.3 .

The authors wish to express their thanks to the referee for pointing out Theorem 3.4 to them and some mistakes in the manuscripts.

\section{REFERENCES}

1. K. R. Davidson, Nest algebras, Pitman Research Notes in Mathematics Series 191, Longman Scientific and Technical, Burnt Mill Harlow, Essex, UK, 1988. MR 90f:47062

2. J. A. Erdos, Some results on triangular operators algebras, Amer. J. Math. 89 (1967), 85-93. MR 35:800

3. J. A. Erdos, Operators of finite rank in nest algebras, J. London Math. Soc. 43 (1968), 391397. MR 37:5721

4. R. V. Kadison, Triangular algebras - another chapter, in Selfadjoint and Nonselfadjoint Operator Algebras and Operator Theory (R. S. Dotan, ed.), Contemp. Math., vol. 120, Amer. Math. Soc., 1991, pp. 63-76. MR 92h:47059

5. R. V. Kadison and I. M. Singer, Triangular operator algebras, Amer. J. Math. 82 (1960), 227-259. MR 22:12409 
6. J. L. Orr, On the closure of triangular algebras, Bull. Amer. Math. Soc. 23 (2) (1990), 461467.

7. Y. T. Poon, Maximal triangular subalgebras need not be closed, Proc. Amer. Math. Soc. 111 (2) (1991), 475-479. MR 91f:46082

8. J. R. Ringrose, Algebraic isomorphisms between ordered bases, Amer. J. Math. 83 (1961), 463-478. MR 25:4335

9. J. R. Ringrose, On some algebras of operators, Proc. London Math. Soc. 15 (3) (1965), 61-83. MR 30:1405

10. J. R. Ringrose, On some algebras of operators. II, Proc. London Math. Soc. 3 (3) (1966), 385-402. MR 33:4703

School of Mathematical Science, Suzhou University, Suzhou 215006, People's RepubLIC OF CHINA

E-mail address: fylu@suda.edu.cn

Department of Mathematics, Zhejiang University, Hangzhou 310027, People's RepubLIC OF CHINA 